\title{
The Giant X\#Ray Flare of NGC 5905: Tidal Disruption of a Star, a Brown Dwarf, or a Planet?
}

\section{Citation}

Li, Li\#Xin, Ramesh Narayan, and Kristen Menou. 2002. "The Giant X\#Ray Flare of NGC 5905:

Tidal Disruption of a Star, a Brown Dwarf, or a Planet?" The Astrophysical Journal 576 (2): $753-$ 61. https://doi.org/10.1086/341890.

\section{Permanent link}

http://nrs.harvard.edu/urn-3:HUL.InstRepos:41384945

\section{Terms of Use}

This article was downloaded from Harvard University's DASH repository, and is made available under the terms and conditions applicable to Other Posted Material, as set forth at http:// nrs.harvard.edu/urn-3:HUL.InstRepos:dash.current.terms-of-use\#LAA

\section{Share Your Story}

The Harvard community has made this article openly available.

Please share how this access benefits you. Submit a story.

Accessibility 


\title{
The Giant X-Ray Flare of NGC 5905: Tidal Disruption of a Star, a Brown Dwarf, or a Planet?
}

\author{
Li-Xin Li ${ }^{a, 1}$, Ramesh Narayan ${ }^{a}$, and Kristen Menou ${ }^{b, 1}$ \\ ${ }^{a}$ Harvard-Smithsonian Center for Astrophysics, Cambridge, MA 02138, USA \\ lli,rnarayan@cfa.harvard.edu \\ ${ }^{b}$ Princeton University Observatory, Princeton, NJ 08544, USA \\ kristen@astro.princeton.edu
}

\begin{abstract}
We model the 1990 giant X-ray flare of the quiescent galaxy NGC 5905 as the tidal disruption of a star by a supermassive black hole. From the observed rapid decline of the luminosity, over a timescale of a few years, we argue that the flare was powered by the fallback of debris rather than subsequent accretion via a thin disk. The fallback model allows constraints to be set on the black hole mass and the mass of debris. The latter must be very much less than a solar mass to explain the very low luminosity of the flare. The observations can be explained either as the partial stripping of the outer layers of a low-mass main sequence star or as the disruption of a brown dwarf or a giant planet. We find that the X-ray emission in the flare must have originated within a small patch rather than over the entire torus of circularized material surrounding the black hole. We suggest that the patch corresponds to the "bright spot" where the stream of returning debris impacts the torus. Interestingly, although the peak luminosity of the flare was highly sub-Eddington, the peak flux from the bright spot was close to the Eddington limit. We speculate on the implications of this result for observations of other flare events.
\end{abstract}

Subject headings: accretion, accretion disks — black hole physics — galaxies: nuclei

\footnotetext{
${ }^{1}$ Chandra Fellow
} 


\section{Introduction}

Tidal disruption of a star by a supermassive black hole in a galactic nucleus has been investigated by many authors. Early seminal investigations by Hills (1975); Lacy, Townes, \& Hollenbach (1982); Rees (1988); Evans \& Kochanek (1989) and Cannizzo, Lee, \& Goodman (1990) were followed up with detailed analytical studies (Kochanek 1994; Khokhlov, \& Melia 1996; Loeb \& Ulmer 1997; Ulmer, Paczyński, \& Goodman 1998; Ulmer 1999) and numerical simulations (Khokhlov, Novikov, \& Pethick 1993; Frolov et al. 1994; Marck, Lioure, \& Bonazzola 1996; Diener et al. 1997; Kim, Park, \& Lee 1999; Ayal, Livio, \& Piran 2000; Ivanov \& Novikov 2001).

A tidal disruption event is expected to produce a luminous flare of electromagnetic radiation in the UV to X-ray band. Komossa (2001) argued that a convincing detection of a tidal disruption event must fulfill the following three criteria: (1) The event should be of short duration (a "flare"); (2) It should be very luminous (up to $L_{\max } \sim 10^{45} \mathrm{erg} \mathrm{s}^{-1}$ at maximum); (3) It should reside in a galaxy that is otherwise non-active (in order to exclude an upward fluctuation in the mass accretion rate of an active nucleus).

ROSAT observations have led to the discovery of four X-ray flaring "normal" galaxies that fulfill the above criteria [summarized in Komossa (2001)]: NGC 5905, RXJ1242-1119, RXJ1624+7554, RXJ1420+5334; plus a possible fifth candidate: RXJ1331-3243. All these galaxies have exhibited large X-ray flares (peak luminosity up to $\sim 10^{44} \mathrm{erg} \mathrm{s}^{-1}$ ), corresponding to a significant amplitude of variability (up to a factor $\sim 200$ ), an ultra-soft X-ray spectrum $\left(k_{B} T_{\mathrm{bb}} \approx 0.04-0.1 \mathrm{keV}\right.$, where $T_{\mathrm{bb}}$ is the temperature derived from a black-body fit to the spectral data), and no signs of Seyfert-like activity in the optical. These flares are promising candidates for tidal disruption events (Komossa 2001). A UV flare has also been detected at the center of a mildly active elliptical galaxy, NGC 4552, with a bolometric luminosity $\sim 10^{39} \mathrm{erg} \mathrm{s}^{-1}$. Since the luminosity is several orders of magnitude less than would be expected from complete tidal disruption of a star, the event has been explained as the tidal stripping of a stellar atmosphere (Renzini et al. 1995).

Among the five galaxies listed above, long-term follow-up observations exist only for NGC 5905 (Komossa \& Bade 1999). In this paper, we model the light curve of NGC 5905 assuming that the flare was produced by the tidal disruption of a star by a supermassive black hole. We compare theoretical predictions with the observational data and constrain the properties of the progenitor star (e.g., its mass and radius) and estimate how much mass was accreted by the black hole. 


\section{The Light Curve of the Flare in NGC 5905}

A giant X-ray flare in NGC 5905 was detected by ROSAT in July 1990 (Bade, Komossa, \& Dahlem 1996). The observed peak X-ray luminosity exceeded $10^{42} \mathrm{erg} \mathrm{s}^{-1}$, and the X-ray spectrum was very soft (photon index $\Gamma_{X} \approx-4$, or a very cool black-body with $k_{B} T_{\mathrm{bb}} \approx 0.06$ $\mathrm{keV}$ ). Follow-up observations were made in 1990 and 1993 with the PSPC, and in 1996 with the HRI (Komossa \& Bade 1999). The observational data are listed in Table 1. Assuming a Hubble constant $H_{0}=50 \mathrm{~km} \mathrm{~s}^{-1} \mathrm{Mpc}^{-1}$, the count rates may be converted to luminosities, which are plotted with error bars in Figure 1. The two downward arrows are upper limits. The light curve after the initial burst gradually declines with time. During the X-ray outburst as well as at later times, the optical brightness is constant to within observational errors $\left(\sim 0.2^{m}\right)$.

Fitting the late decline of the NGC 5905 light curve (the four last data points in Table 1) with a $\left(t-t_{D}\right)^{-5 / 3}$ law that is predicted by the fallback model of tidal disruption (see $\S 3$ ), and treating the largest luminosity observed at $t=1990.54 \mathrm{yr}$, viz. $L_{X}=4.47 \times 10^{42} \mathrm{erg} \mathrm{s}^{-1}$, as a lower limit (since this was still in the rising part of the light curve), we obtain

$$
L_{X}=(0.30 \pm 0.03) \times 10^{42} \mathrm{erg} \mathrm{s}^{-1}\left[\frac{t-(1990.36 \pm 0.02) \mathrm{yr}}{1 \mathrm{yr}}\right]^{-5 / 3}
$$

where the time $t$ is in units of years, and $t_{D}=1990.36 \pm 0.02 \mathrm{yr}$ is the moment when tidal disruption happened.

During the first epoch of observations at time $t=1990.54$, the X-ray flux rose steadily (see Table 1), strongly suggesting that this epoch corresponded to the onset of the flare. For a start time of $t_{1} \approx 1990.54 \mathrm{yr}$, the total X-ray energy released during the entire outburst event is

$$
\Delta E_{X}=\int_{t_{1}}^{\infty} L_{X}(t) d t \approx(4.5 \pm 0.9) \times 10^{49} \mathrm{erg}
$$

A black-body fit to the X-ray spectrum during the outburst gives a temperature $k_{B} T_{\mathrm{bb}} \approx$ $0.06 \mathrm{keV}$, and an intrinsic $(0.1-2.4 \mathrm{keV})$ mean luminosity $L_{X} \approx 3 \times 10^{42} \mathrm{erg} \mathrm{s}^{-1}$. Integrating the black-body spectrum over all energies (from zero to infinity), the bolometric luminosity is $L \approx 1.1 L_{X}$. Thus, if the spectrum does indeed have a black-body form, the bolometric correction is not very significant. Then, the total mass associated with the released energy may be estimated to be

$$
\Delta M=\frac{\Delta E}{\epsilon c^{2}} \approx \frac{\Delta E_{X}}{\epsilon c^{2}} \approx(2.5 \pm 0.5) \times 10^{-4} M_{\odot}\left(\frac{\epsilon}{0.1}\right)^{-1}
$$


where $\epsilon$ is the efficiency of converting mass to radiated energy. This mass estimate is similar to that for the UV flare in NGC 4552 (Renzini et al. 1995). Note that our estimate of $\Delta E_{X}$ depends only weakly on the assumed power law index $5 / 3$ in equation (1). This is because the observed light curve extends over a long enough time to capture most of the emission in the flare. The estimate of $\Delta M$ suffers from the uncertainty in the bolometric correction (which we have taken to be unity).

In models of thin accretion disks around black holes, the color temperature $T_{\mathrm{bb}}$ of the emitted radiation is generally higher than the effective temperature $T_{\text {eff }}$, defined by flux $F=\sigma T_{\text {eff }}^{4}$. The deviation of $T_{\mathrm{bb}}$ from $T_{\text {eff }}$ is caused by electron scattering and Comptonization (Ross, Fabian \& Mineshige 1992; Shimura \& Takahara 1993, 1995a, 1995b). Writing $T_{\mathrm{bb}}=$ $f_{c} T_{\text {eff }}$, the factor $f_{c}$ can be estimated from detailed self-consistent models of the radiative transfer in the disk. It is found that the value of $f_{c}$ depends on both the mass of the black hole (compare Shimura \& Takahara 1993 and 1995b) and the mass accretion rate. Shimura \& Takahara (1993) present results for a $10^{8} M_{\odot}$ black hole emitting at a radius of 5 Schwarzschild radii, which is similar to the conditions we expect in our problem. Depending on the accretion rate, they obtain values of $f_{c}$ in the range $1.3-3.2$ (see their Fig. 4 and the associated discussion), with the highest value of $f_{c}$ being obtained for near-Eddington rate accretion. In what follows, we retain the factor $f_{c}$ in the equations, and we substitute a value of 3 whenever we require a numerical estimate.

Including the factor $f_{c}$ as defined above, we estimate the radius of the X-ray emitting region of the flare in NGC 5905 to be

$$
R_{X}=\left(\frac{f_{c}^{4} L_{X}}{\pi \sigma T_{\mathrm{bb}}^{4}}\right)^{1 / 2} \approx 2.4 \times 10^{12} \mathrm{~cm}\left(\frac{f_{c}}{3}\right)^{2}, \quad f_{c} \equiv \frac{T_{\mathrm{bb}}}{T_{\mathrm{eff}}}
$$

where we have used $L_{X} \approx 3 \times 10^{42} \mathrm{erg} \mathrm{s}^{-1}$ for the mean luminosity during the peak of the outburst (Komossa 2001). The radius we derive is surprisingly small; even after setting $f_{c}=3$, it is barely equal to the Schwarzschild radius of a $10^{7} M_{\odot}$ black hole, whereas an accretion disk is likely to have an effective radiating area $10-100$ times larger. We return to this point in $\S 5$.

\section{Tidal Disruption of a Star by a Supermassive Black Hole}

The tidal disruption radius of a star in the vicinity of a Schwarzschild black hole is

$$
r_{T}=\mu R_{\star}\left(\frac{M_{H}}{M_{\star}}\right)^{1 / 3}
$$


where $M_{H}$ is the mass of the black hole, $M_{\star}$ is the mass of the star, $R_{\star}$ is the radius of the star, and $\mu$ is a dimensionless coefficient. The exact value of $\mu$ is uncertain, though physically we expect it to be of order unity. We assume $\mu=1$ in our modeling.

The ratio of $r_{T}$ to $r_{H}$, where $r_{H}=2 G M_{H} / c^{2}$ is the Schwarzschild radius of the black hole, is

$$
\frac{r_{T}}{r_{H}}=5.08\left(\frac{M_{H}}{10^{7} M_{\odot}}\right)^{-2 / 3}\left(\frac{M_{\star}}{M_{\odot}}\right)^{-1 / 3}\left(\frac{R_{\star}}{R_{\odot}}\right) .
$$

For a solar-type star with $M_{\star}=M_{\odot}$ and $R_{\star}=R_{\odot}$, we have $r_{T}<r_{H}$ when $M_{H}>1.1 \times$ $10^{8} M_{\odot}$. In such a case the star will be swallowed whole by the black hole, without a tidal disruption event (Hills 1975).

After a star is tidally disrupted, there are two distinct stages in the dynamical evolution of the debris, each with its own time development and luminosity:

(1) The fallback stage (Rees 1988): A fraction of the material in the disrupted star remains gravitationally bound to the black hole and falls back to the pericenter, giving rise to a mass accretion rate (and a luminosity) evolving with time as $\sim t^{-5 / 3}$ (Phinney 1989). The returning material shocks and then circularizes to form an orbiting torus around the black hole at a radius $r_{c}=2 r_{P}$ (by angular momentum conservation), where $r_{P}$ is the pericentric radius. The total energy radiated during the fallback is given by $G M_{H} \Delta M / 2 r_{c}$, where $\Delta M$ is the fallback mass. This energy can be less than the energy released in the subsequent viscous accretion stage discussed below. Nevertheless, since the fallback stage is relatively short-lived, it dominates the early luminosity of a disruption event.

(2) The viscous accretion stage (Cannizzo, Lee, \& Goodman 1990): The torus formed from returning debris gradually spreads inward and outward from $r=r_{c}$ under the action of viscosity, and gives rise to a mass accretion rate (and a luminosity) evolving with time approximately as $\sim t^{-1.2}$. The energy radiated during this stage is given by $G M_{\mathrm{H}} \Delta M\left[\left(1 / 2 r_{m s}\right)-\right.$ $\left(1 / 2 r_{c}\right)$ ], where $r_{m s}$ is the radius of the last stable circular orbit (=3 Schwarzschild radii for a non-spinning black hole). The accretion occurs on a viscous time scale, which is typically very long compared to the fallback time (Cannizo et al. 1990; Ulmer 1999; Appendix A of the present paper). Therefore, this stage is expected to last a long time, up to hundreds of years, depending on the magnitude of the viscosity in the disk, and the luminosity is significantly lower than that associated with the fallback stage (Appendix A).

In the case of NGC 5905, the X-ray luminosity was seen to rise rapidly during the first epoch of observations at $t=1990.54 \mathrm{yr}$, indicating that the flare probably began around that time (see Table 1 and Fig. 1). The luminosity then dropped by more than a factor of 100 between $t=1990.54 \mathrm{yr}$ and $t=1996.89 \mathrm{yr}$. Such a rapid decline indicates that the 
observed outburst of NGC 5905 could not have been due to the accretion phase, but must have corresponded to the fallback stage (if it indeed was a tidal disruption event). Therefore, in this paper we assume that the flare in NGC 5905 corresponds to the fallback stage of tidal disruption.

Let us assume that the center of the star follows a nearly parabolic orbit (i.e., the binding energy of the star to the black hole is close to zero), and for definiteness let us assume that the pericenter of the orbit is at $r_{P}=r_{T}$, i.e. that the parameter $\eta$ defined below in equation (8) is equal to unity. Since the specific energy at the center of the star is $E_{c}=v_{P}^{2} / 2-G M_{H} / r_{T}=0$, the orbital velocity of the star at pericenter is $v_{P}=\left(2 G M_{H} / r_{T}\right)^{1 / 2}=c\left(r_{H} / r_{T}\right)^{1 / 2}$, and the spread in the specific energy of the disrupted debris, $2 \Delta E$, is governed by the variation of the black hole gravitational potential across the star, and the spin-up of the star as a result of the tidal interaction (Rees 1988). If $M_{H} \gg M_{\star}$, we have

$$
\Delta E=k \frac{G M_{\star}}{R_{\star}}\left(\frac{M_{H}}{M_{\star}}\right)^{1 / 3},
$$

where $k$ depends on the spin-up state of the star. If the star is spun up to the break-up spin angular velocity, we have $k \approx 3$. On the other hand, if the spin-up effect is negligible, then we have $k \approx 1$ (Rees 1988; Lacy, Townes, \& Hollenbach 1982; Evans \& Kochanek 1989; Ayal, Livio, \& Piran 2000). Since the spin-up velocity is always $\ll v_{P}$, the energy used to spin up the star is much less than the orbital kinetic energy of the star, and the stellar orbit remains parabolic to a very good approximation.

The spin-up of a star via tidal interaction is a complex process. In linear perturbation theory, the spin-up angular velocity is given by (Press \& Teukolsky 1977; Alexander \& Kumar 2001; Alexander \& Livio 2001)

$$
\omega_{s} \approx \frac{T_{2}(\eta)}{2 \alpha \eta^{2}} \omega_{P}, \quad \eta \equiv\left(\frac{r_{P}}{r_{T}}\right)^{3 / 2},
$$

where $\eta \approx 1$ in our problem, $\omega_{P} \equiv v_{P} / r_{P}$ is the orbital angular velocity of the star at the pericenter, $\alpha$ is the stellar momentum of inertia in units of $M_{\star} R_{\star}^{2}$, and $T_{2}$ is the second tidal coupling coefficient which is a function of $\eta$. In equation (8) we have assumed that $M_{H} \gg M_{\star}$. For a main sequence star of mass $0.76 M_{\odot}$ and radius $0.75 R_{\odot}$, Alexander \& Kumar (2001) give $\alpha \approx 0.07$ and $T_{2}(1) \approx 0.06$, which implies that $\omega_{s} \approx 0.43 \omega_{P}$ if $\eta=1$. For an $n=1.5$ polytrope star, they give $\alpha \approx 0.21$ and $T_{2}(1) \approx 0.36$, which corresponds to $\omega_{s} \approx 0.86 \omega_{P}$. Furthermore, they show that numerical simulations that include non-linear effects lead to a larger energy transfer from the orbit to the star and a larger spin-up than predicted by linear theory. For these reasons, we feel that it is likely a tidally disrupted star will be spun up to nearly the break-up limit, with $\omega_{s} \approx \omega_{P}$ and $k \approx 3$. We therefore assume 
$k=3$ for most of our analysis; however, for completeness we also present a few results for the non-spinning case $k=1$.

Part of the disrupted star has positive energy and escapes from the system, and part remains bound. The bound material follows a highly eccentric orbit and returns to the central black hole after completing one orbit. The flare begins when the most bound material, with specific energy $E_{\min }=-\Delta E$, returns. The time since disruption for this to happen is given by

$$
\Delta t_{1}=2 \pi G M_{H}(2 \Delta E)^{-3 / 2} \approx 0.068 \mathrm{yr}\left(\frac{M_{H}}{10^{7} M_{\odot}}\right)^{1 / 2}\left(\frac{M_{\star}}{M_{\odot}}\right)^{-1}\left(\frac{R_{\star}}{R_{\odot}}\right)^{3 / 2}
$$

where we have used $r_{P}=r_{T}$ and $k=3^{2}$. Material that is less bound takes a progressively longer time to return, and the overall light curve is determined by the distribution of debris mass as a function of binding energy.

Rees (1988) assumed that the debris is uniformly distributed in mass between $-\Delta E$ and $+\Delta E$. Numerical simulations (Evans \& Kochanek 1989; Ayal, Livio, \& Piran 2000) have shown that this is a reasonable approximation. The bound material then returns to pericenter at the rate (Phinney 1989; Evans \& Kochanek 1989)

$$
\dot{M}=\frac{2 \Delta M}{3 \Delta t_{1}}\left(\frac{t-t_{D}}{\Delta t_{1}}\right)^{-5 / 3}=A\left(\frac{t-t_{D}}{1 \mathrm{yr}}\right)^{-5 / 3} \quad \Delta M \equiv f M_{\star},
$$

where $t_{D}$ is the time of the initial tidal disruption, $\Delta M$ is the actual mass that falls back to pericenter, which is a fraction $f$ of the original mass of the star, and

$$
A \approx 7.0 \times 10^{23} \mathrm{~g} \mathrm{~s}^{-1}\left(\frac{f}{0.1}\right)\left(\frac{M_{H}}{10^{7} M_{\odot}}\right)^{1 / 3}\left(\frac{M_{\star}}{M_{\odot}}\right)^{1 / 3}\left(\frac{R_{\star}}{R_{\odot}}\right) .
$$

In Rees' original model, the whole star is disrupted and half the debris is on bound orbits, so that $f=0.5$. However, a recent numerical simulation shows that not all the returning material is captured by the black hole: about $75 \%$ of the returning mass becomes unbound following the large compression it experiences on the way back (Ayal, Livio, \& Piran 2000). This gives rise to a smaller $f \approx 0.1$. Another possibility giving rise to a small $f$ is that the star is only partially disrupted: its envelope could be stripped by the black hole, leaving most of its core nearly intact (Renzini et al. 1995; see also $§ 5$ below). Here we treat $f$ as a free parameter.

\footnotetext{
${ }^{2}$ If the star is on a parabolic orbit with $r_{P}<r_{T}$, then the spin-up effect will be less important and we will have a smaller $k$ (but $k$ is still $\gtrsim 1$ ), which will give rise to a $\Delta t_{1}$ that is somewhat larger than that given by eq. (9).
} 
The gravitational potential energy available from fallback is determined by the difference between the specific binding energy of the circularization orbit at $r=2 r_{P}=2 r_{T}$ and the specific binding energy of the incoming material. Since $M_{H} \gg M_{\star}$, all the bound debris is on highly eccentric orbits with a specific binding energy much smaller than the binding energy of the final circular orbit. Thus, assuming that the fallback material radiates the energy release promptly, the radiation efficiency $\epsilon$ is independent of time during fallback:

$$
\epsilon \approx \frac{G M_{\star}}{4 R_{\star} c^{2}}\left(\frac{M_{H}}{M_{\star}}\right)^{2 / 3} \approx 0.025\left(\frac{M_{H}}{10^{7} M_{\odot}}\right)^{2 / 3}\left(\frac{M_{\star}}{M_{\odot}}\right)^{1 / 3}\left(\frac{R_{\star}}{R_{\odot}}\right)^{-1} .
$$

The luminosity of the fallback process is then given by

$$
L=\epsilon \dot{M} c^{2} \approx 1.55 \times 10^{43} \operatorname{erg~s}^{-1}\left(\frac{f}{0.1}\right)\left(\frac{M_{H}}{10^{7} M_{\odot}}\right)\left(\frac{M_{\star}}{M_{\odot}}\right)^{2 / 3}\left(\frac{t-t_{D}}{1 \mathrm{yr}}\right)^{-5 / 3} .
$$

The luminosity peaks at $t=t_{D}+\Delta t_{1}$, i.e. when the most bound debris falls back to the pericenter, so we have

$$
L_{\text {peak }} \approx 1.36 \times 10^{45} \mathrm{erg} \mathrm{s}^{-1}\left(\frac{f}{0.1}\right)\left(\frac{M_{H}}{10^{7} M_{\odot}}\right)^{1 / 6}\left(\frac{M_{\star}}{M_{\odot}}\right)^{7 / 3}\left(\frac{R_{\star}}{R_{\odot}}\right)^{-5 / 2} .
$$

\section{Comparison with the Flare in NGC 5905}

In $\S 2$ we have fitted the decline of the NGC 5905 light curve to a $\left(t-t_{D}\right)^{-5 / 3}$ law, and obtained the result given in equation (1) with a disruption time of $t_{D}=1990.36 \mathrm{yr}$. The fit is acceptable (see Fig. 1), and the time at which the light curve reached its peak is fairly well constrained. Since the initial measurements at $t \approx 1990.54 \mathrm{yr}$ showed flux rising with time, the maximum in the light curve was clearly later than this time. At the same time, the maximum could not have been later than $1990.56 \mathrm{yr}$, since the fitted light curve in (1) falls below $L \approx 4.47 \times 10^{42} \mathrm{erg} \mathrm{s}^{-1}$ at that time. Thus, the light curve fit gives $\Delta t_{1}$ within the range 0.18 to $0.20 \mathrm{yr}$, where $\Delta t_{1}$ is the time lag between the peak of the light curve and the moment of tidal disruption. However, there is an error of $\pm 0.03 \mathrm{yr}$ on each limit. Therefore, we use conservative bounds on $\Delta t_{1}$

$$
0.15 \mathrm{yr}<\Delta t_{1}<0.23 \mathrm{yr}
$$

¿From the discussion in $\S 2$, the mass involved in the outburst of NGC 5905 is only $\sim 10^{-4} M_{\odot}$. We therefore consider the following two possibilities for the candidate stellar object disrupted in NGC 5905: 
(1) A low mass star (LMS), whose radius and mass are related by $R_{\star}=R_{\odot}\left(M_{\star} / M_{\odot}\right)$ $\left(0.08 M_{\odot}<M_{\star}<1 M_{\odot}\right)$.

(2) A substellar objects (SSOs, including brown dwarfs and planets), whose radius and mass are related by $R_{\star}=0.06 R_{\odot}\left(M_{\star} / M_{\odot}\right)^{-1 / 8}\left(0.01 M_{\odot}<M_{\star}<0.08 M_{\odot}\right)$ (Chabrier \& Baraffe $2000)^{3}$.

Substituting these two radius-mass relations into equation (9) and noting the limits on $\Delta t_{1}$ in equation (15), we find that the black hole mass in NGC 5905 and the mass of the disrupted star are restricted to the two relatively narrow shaded bands shown in Figure 2. The figure makes use of an additional constraint, namely a lower limit on the tidal radius $r_{T}$. If the black hole is not rotating, then the smallest distance to which a star on a parabolic orbit can approach and yet not be captured by the black hole is $r=4 r_{g}$, where $r_{g} \equiv G M_{H} / c^{2}=r_{H} / 2$. If we have a maximally rotating black hole and if the angular momentum of the stellar orbit is parallel to the spin of the black hole, then the closest approach possible is $r=r_{g}$. The real situation is probably in between these two limits, so we use a fiducial limiting radius of $r=2 r_{g}$. Using equation (6), we have calculated relations connecting $M_{H}$ and $M_{\star}$ for all three limiting radii and plotted them as dotted lines in Figure 2.

The mass of the black hole in NGC 5905 is not very well constrained, but it is believed to be in the range $M_{H} \sim 10^{7}-10^{8} M_{\odot}$ (Komossa 2001), based on the correlation between bulge blue luminosity and black hole mass for spiral galaxies (Salucci et al. 2000) and on the correlation between bulge velocity dispersion and black hole mass for ellipticals and spirals (Gebhardt et al. 2000; Merritt \& Ferrarese 2001). This information is sufficient to constrain the mass of the disrupted star fairly tightly: the mass must be either in the range $0.01-0.02 M_{\odot}$ in the case of a SSO or $0.6-1 M_{\odot}$ in the case of a LMS.

The result, however, depends sensitively on the choice we make for the tidal spin-up parameter $k$ discussed in $\S 2$. Figure 2 has been calculated for $k=3$, a reasonable and possibly likely value, but in principle $k$ could be as small as 1 . Figure 3 shows how the mass constraints change when we use the latter value. We see that the allowed range of models is limited to somewhat lower black hole masses, unless the black hole spins very rapidly and allows tidal disruptions down to $r_{T} \sim r_{g}$. We do not discuss the $k=1$ case further.

\footnotetext{
${ }^{3}$ Chabrier \& Baraffe (2000) state that their fit is good down to $M_{\star}=0.01 M_{\odot}$, but a comparison with their plot shows that the fit is acceptable even for lower masses. We apply their scaling down to $M_{\star} \sim 0.006 M_{\odot}$.
} 
¿From equations (13) and (1) we infer that

$$
\frac{M_{H}}{10^{7} M_{\odot}}\left(\frac{M_{\star}}{M_{\odot}}\right)^{2 / 3}=\frac{0.0019}{f},
$$

where $f$ is the fraction of the mass of the star that returns as fallback debris. The dashed lines in Figure 2 correspond to $f=0.0005,0.012$, and 0.12 respectively. We see that, for the case of a LMS, $f$ must be $<0.0005$, which means that an unusually small fraction of the star must have participated in the fallback. For the case of a SSO, somewhat larger values of $f$ are obtained (but still rather small).

In Figures 4 and 5 we plot the luminosities given by equation (13) for different values of $M_{H}, M_{\star}$, and $f$, where the disrupted object is assumed to be a LMS (Fig. 4) and a SSO (Fig. 5), respectively, and the disruption time is taken to be $t_{D}=1990.36 \mathrm{yr}$. We have chosen values of $\left(M_{H}, M_{\star}\right)$ from the shaded regions in Figure 2 so that equation (15) and the

condition $r_{T}>2 r_{g}$ are satisfied, and chosen $f$ such that $L_{\text {peak }} \gtrsim 4.47 \times 10^{42} \mathrm{erg} \mathrm{s}^{-1}$ where $L_{\text {peak }} \equiv L\left(t=t_{D}+\Delta t_{1}\right)$ is the peak luminosity.

We confirm that, in order for the models to fit the observational data, a very small $f$ $(<0.0003)$ is required for the case of a LMS (Fig. 4). For the case of a SSO (Fig. 5) a fairly small $f(\lesssim 0.04)$ is again required if $M_{H} \gtrsim 2 \times 10^{7} M_{\odot}$. A largish $f(>0.1)$ can be obtained only if the black hole has a very small mass: $M_{H}<4 \times 10^{6} M_{\odot}$.

In Figures 4 and 5 we also indicate the ratio of the peak flux, $F_{\text {peak }}$, in the fitted light curve to the Eddington flux, $F_{\text {Edd }}$, where $F_{\text {peak }} \equiv L_{\text {peak }} /\left(\pi R_{X}^{2}\right)$ with $R_{X}$ given by equation (4), and $F_{\text {Edd }} \equiv L_{\text {Edd }} /\left(4 \pi r_{T}^{2}\right)$, with $L_{\text {Edd }}=1.3 \times 10^{45} \mathrm{erg} \mathrm{s}^{-1}\left(M_{H} / 10^{7} M_{\odot}\right)$ and $r_{T}$ given by equation (6). We see that the ratio $F_{\text {peak }} / F_{\text {Edd }}$ is significantly larger than unity if the color temperature factor $f_{c} \sim 1$, and it is not much less than unity even if $f_{c} \sim 3$ (the likely value). We discuss the implications of this result in the next section.

\section{Summary and Discussion}

The X-ray light curve of the flare in NGC 5905 agrees with the $\left(t-t_{D}\right)^{-5 / 3}$ dependence predicted for fallback of debris after tidal disruption of a star by a supermassive black hole (Fig. 1). If the black-body fit presented in Bade et al. (1996) is a good representation of the spectrum of the emission, then the bolometric correction to the X-ray flux is not very large and the observed light curve gives a meaningful measure of the energetics and luminosity of the flare. We note that, during and after the X-ray outburst, the optical brightness did not show any significant variation (Komossa \& Bade 1999). This confirms that the bulk of the fallback emission probably did occur in X-rays. (If the flare had substantial hidden emission 
in the far ultraviolet band, it is hard to see how there could have been no variations in the optical.)

In addition to fallback, a second independent stage of evolution is expected when the circularized fallback material viscously accretes onto the black hole. This stage is expected to develop much more slowly and to be very much dimmer than the fallback stage (Appendix A). It is not relevant for the particular flare in NGC 5905 considered here.

The time lag between the estimated disruption time $t_{D}=1990.36 \mathrm{yr}$ of the event and the time $t_{1}$ corresponding to the peak of the light curve gives the orbital period $\Delta t_{1}$ of the most tightly bound debris. The data constrain $\Delta t_{1}$ fairly tightly (eq. [15]). By the arguments described in $\S 3$, this translates into constraints on the mass $M_{H}$ of the black hole and the mass $M_{\star}$ of the disrupted star. The constraints are shown in Figure 2.

In deriving equation (9), we have estimated the binding energy of the most tightly bound debris assuming that the star is fully spun up by tidal interaction before being disrupted. This corresponds to a spin-up parameter $k=3(\S 3)$; this value is supported both by linear analysis (Press \& Teukolsky 1977) and numerical simulations (Alexander \& Kumar 2001). If on the other hand there is no tidal spin-up, then we expect $k=1$, and the results change significantly (Fig. 3). We consider $k=1$ to be somewhat unlikely, and have focused on the $k=3$ results.

There is also ambiguity in the value of the parameter $\mu$ in the definition of $r_{T}$ [eq. (5)]. Numerical simulations show that $\mu$ is usually of order unity, the value we use, but that it can be as large as 1.7 in specific cases (Frolov et al. 1994; Diener et al. 1997). The time interval $\Delta t_{1}$ [eq. (9)] is very sensitive to the value of $\mu: \Delta t_{1} \propto \mu^{3}$. Because of the tight observational constraint on $\Delta t_{1}$ (Figs. 2 and 3), $\mu$ cannot be much larger than 1 since otherwise it would imply too small a black hole mass.

Another constraint on $M_{H}$ and $M_{\star}$ is obtained from the requirement that the radius $r_{T}$ at which the star is tidally disrupted must lie outside the radius of the marginally bound circular orbit. This requires $r_{T}>4 r_{g}$ for a Schwarzschild black hole and $r_{T}>r_{g}$ for the most favorably oriented orbit around a maximally spinning Kerr hole. The uncertainty in the limiting $r_{T}$ is indicated by the dotted lines in Figure 2. For concreteness, we have assumed that the limiting radius is intermediate between the two extreme cases mentioned above; we have used the limit $r_{T}>2 r_{g}$. Yet another source of uncertainty derives from the fact that we have used Newtonian dynamics in our analysis, whereas it is clear that relativistic effects should be important (Luminet \& Marck 1985; Laguna et al. 1993; Frolov et al. 1994; Diener et al. 1997).

Ignoring these caveats, we obtain a tight correlation between the mass of the disrupted 
star in NGC 5905 and the mass of the central black hole. As Figure 2 shows, the data are consistent with two distinct solution branches.

One solution branch corresponds to a relatively massive black hole, $M_{H}$ close to $10^{8} M_{\odot}$, tidally disrupting a main sequence star with $M_{\star} \sim M_{\odot}$. The other branch consists of a lower mass black hole, $M_{H}<10^{7.5} M_{\odot}$, disrupting a brown dwarf or a giant planet with $M_{\star} \lesssim 0.02 M_{\odot}$. Assuming that NGC 5905 satisfies the well-known correlations between black hole mass and host galaxy properties (Salucci et al. 2000; Gebhardt et al. 2000; Merritt \& Ferrarese 2001), the black hole mass is estimated to be $M_{H} \sim 10^{7}-10^{8} M_{\odot}$ (Komossa 2001). This mass range permits both solution branches.

Figures 4 and 5 show sample theoretical light curves corresponding to the two solution branches. In each case, there is one free parameter, namely the fraction $f$ of the mass of the disrupted star that returns as fallback debris to produce the observed radiation. We find that $f$ is unusually small if the disrupted star is a main sequence star; for the particular example shown in Figure 4, we require $f<0.0003$, which is an extremely small value compared to the theoretically expected value of $f \sim 0.1-0.5$. Brown dwarf models give somewhat larger estimates for $f$ (Fig. 5), though still uncomfortably small, unless the black hole has an unusually low mass $\left(<10^{6.5} M_{\odot}\right)$. Though the estimated $f$ can be increased by adopting a larger ambiguity factor $\mu$ in the definition of $r_{T}$, we do not consider this likely since a larger $\mu$ will lead to an unacceptably small value of $M_{H}$.

The above results on $f$ are not surprising. As we showed in $\S 2$, the total radiative energy in the flare is only $4 \times 10^{49} \mathrm{erg}$, which for any reasonable radiative efficiency corresponds to an unexpectedly small mass $\sim$ few $\times 10^{-4} M_{\odot}$. Figures 4 and 5 merely confirm this result using a detailed estimate of the radiative efficiency (eq. 12).

Can $f$ be so small for a tidally disrupted solar mass star? Rees (1988) and Renzini et al. (1995) have argued that a star could lose just its outer layers if the minimum distance from the star to the black hole is somewhat larger than $r_{T}$. Here we point out a related possibility. The usual formula for the tidal disruption radius, $r_{T}=R_{\star}\left(M_{H} / M_{\star}\right)^{1 / 3}$, is correct only for a homogeneous star with a uniform mass density. For a real star, we could define a variable tidal disruption radius,

$$
r_{T}(R)=R\left[\frac{M_{H}}{M(R)}\right]^{1 / 3}=\left[\frac{3 M_{H}}{4 \pi \bar{\rho}(R)}\right]^{1 / 3},
$$

where $R \leq R_{\star}$ is a radius inside the star, $M(R)$ is the mass contained within $R$, and $\bar{\rho}(R)$ is the corresponding mean density. The meaning of $r_{T}(R)$ is as follows: if the star is located at a distance $r=r_{T}(R)$ from the black hole, then the part of the star outside radius $R$ (i.e., the spherical envelope between $R$ and $R_{\star}$ ) is tidally stripped off, and the core inside 
$R$ remains intact. Obviously, $r_{T, \max }=r_{T}\left(R_{\star}\right)$ and $r_{T, \min }=\left(3 M_{H} / 4 \pi \rho_{c}\right)^{1 / 3}$, where $\rho_{c}$ is the central density of the star. If the star is at a distance $r$ from the black hole such that $r_{T, \min }<r<r_{T, \max }$, then only a part of the envelope of the star is peeled off.

One important question is how the core of the star responds when it loses its envelope. What is relevant is the response on a dynamical time. Webbink (1985) has calculated the logarithmic derivative of the radius of a main sequence star with respect to mass, $\zeta_{s} \equiv$ $\left(\partial \ln R_{\star} / \partial \ln M_{\star}\right)_{s}$, where the subscript $s$ indicates that the entropy profile of the star is held fixed (as appropriate for a dynamical process). Consider a star that loses a fraction of its mass as a result of a tidal interaction and assume that it dynamically adjusts to a new equilibrium corresponding to its reduced mass. If $\zeta_{s}>1 / 3$, then the mean density of the new configuration will be higher than the original mean density of the star. The leftover mass is then stable to further stripping. However, if $\zeta_{s}<1 / 3$, the new configuration will be prone to further tidal mass loss, and one has a runaway situation in which the entire star is likely to be disrupted.

Webbink (1985) shows that $\zeta_{s}>1 / 3$ for $\log \left(M_{\star} / M_{\odot}\right) \gtrsim-0.2$. Since this is precisely the mass range of interest if the disrupted star in NGC 5905 was a main sequence star (Fig. 2), we conclude that partial stripping is a viable possibility. To estimate what fraction of its mass the star might lose, consider a star like the Sun as an example. For the Sun, the mass inside a radius $R_{1}=0.7 R_{\odot}$ is $0.975 M_{\odot}$, and correspondingly, $r_{T}\left(R_{1}\right)=0.706 r_{T, \max }$. Thus, for a range of distances between $r_{T, \max }$ and $0.7 r_{T, \max }$ (a non-negligible range), such a star will lose only a couple of percent of its mass. If we assume that $10 \%$ of this mass returns via fallback, then we can have $f$ as small as $\sim 0.002$. This is still much larger than the value needed to explain the flare in NGC 5905, but it is at least within striking distance.

Fully convective lower main sequence stars and degenerate brown dwarfs have $\zeta_{s} \sim-1 / 3$. These stars are likely to be fully disrupted in tidal encounters since they expand to a larger radius as they lose mass.

Apart from the small value of $f$, another unexpected result of our analysis is that the peak radiative flux emitted during the flare, $F_{\text {peak }}=\sigma T_{\text {eff }}^{4}=\sigma\left(T_{\mathrm{bb}} / f_{c}\right)^{4}$, is close to the Eddington flux. (Here $T_{\mathrm{bb}}$ is the black-body temperature of the radiation and $f_{c}$ is a color correction factor.) The results are shown for our models in Figures 4 and 5 . Even with $f_{c} \sim 3(\S 2)$, we find $F_{\text {peak }} / F_{\text {Edd }} \sim 0.1-0.3$; the value increases rapidly with decreasing $f_{c}$ (as $\left.f_{c}^{-4}\right)$. What makes this surprising is that the luminosity of the source, even at the peak of the flare, is much below the Eddington luminosity: compare $L_{\text {peak }} \sim 4.5 \times 10^{42} \mathrm{erg} \mathrm{s}^{-1}$ with $L_{\text {Edd }} \sim 10^{45}\left(M_{H} / 10^{7} M_{\odot}\right)$ erg s${ }^{-1}$. Clearly, the radiation in the flare must have been emitted from a small patch on the source, and the patch must have projected a small solid angle at the central black hole. This result was anticipated in the model-independent analysis of $\S 2$, 
where we showed that the effective size of the emitting patch is small compared to the area we might expect if the entire accretion disk out to a few $r_{g}$ is involved in the emission.

The small size of the emitting region can be understood in analogy with mass transfer binary stars. In the latter systems, it is known that the cold gas stream from the secondary star impacts the accretion disk at a compact "bright spot" on the outer edge of the disk. Most of the radial kinetic energy of the stream is converted to thermal energy near this spot and radiated locally. The analogy with the tidal disruption problem is close because the returning material after a disruption event comes in similarly as a narrow cold stream (Kochanek 1994; Kim, Park, \& Lee 1999). It is therefore natural that the radiation during the fallback stage should be emitted from a relatively small patch on the source. The effect is probably even more severe in the tidal disruption problem. Lense-Thirring precession may well cause the incoming stream to be misaligned with respect to the orbital plane of the accretion disk (Kochanek 1994), causing the geometric area of the impact spot to become smaller. (We note that in binaries the stream appears to skim over the surface of the disk after impacting at the bright spot, thereby increasing the effective area over which the kinetic energy of the stream is dissipated. This effect would be reduced if the orbit of the stream is not in the plane of the disk.)

Having decided that emission from a small patch is natural for this problem, we should next ask whether it is a pure accident that the flux from the patch in the NGC 5905 flare happens to be nearly equal to the Eddington flux. We would like to suggest that it is perhaps not an accident.

Imagine a narrow stream with a mass accretion rate $\dot{M}$ impacting on the accretion torus at a radius $r_{T}$ and over a small patch that subtends a solid angle $\Delta \Omega_{\text {stream }}$ at the black hole. Let us suppose that the kinetic energy in the stream is thermalized and radiated immediately from the patch. The radiated flux is locally given by $\epsilon \dot{M} c^{2} / \Delta \Omega_{\text {stream }} r_{T}^{2}$. What would happen if this flux is greater than the local $F_{\text {Edd }}$ ? Jeremy Goodman (private communication) suggests that the super-Eddington flux would cause the local radiating atmosphere to puff up and that the radiation would be emitted from a larger patch than the original impact region. Moreover, it would be natural for the radiating patch to self-adjust so that the flux is close to the Eddington limit. According to this picture then, the radius $R_{X}$ estimated in equation (4) refers to the size of the puffed up atmosphere, while the actual transverse size of the stream is smaller. If the stream is extremely narrow, then the flux would be close to Eddington during a significant fraction of the light curve. Equivalently, the black-body temperature of the radiation would remain constant, even while the luminosity decreases.

This picture (which is predicated on the assumption of a narrow fallback stream) suggests that most tidal disruption events should be visible in soft X-rays; the condition 
$F=F_{\text {Edd }}$, coupled with $f_{c} \sim 3$ (see $\S 2$ ), ensures that the blackbody temperature of the radiation will be $\gtrsim 50 \mathrm{eV}$ for a $10^{7} M_{\odot}$ black hole, which is well-matched to X-ray observations. Soft X-ray surveys should thus be well-suited for an investigation of both the frequency of tidal disruption events and the amount of mass accreted in each event. In this connection, it is interesting that, with the exception of the UV flare of Renzini et al. (1995), all known candidate flares for tidal disruption events have been identified in the X-ray band (see Komossa 2001 for five candidates and Choi et al. 2002 for a possible sixth candidate).

Menou \& Quataert (2001) have emphasized that, given current estimates of the rate of tidal disruptions, the nuclei of many nearby normal galaxies should be much brighter than observed if a fair fraction of a stellar mass is accreted by the black hole after each tidal disruption event. This is not relevant for the late accretion phase of the particular NGC 5905 flare discussed here because, as we have discussed (see also the Appendix below), very little mass $\left(\ll 1 M_{\odot}\right)$ returned to the $\mathrm{BH}$ in this case. However, whether we attribute the small amount of mass in the NGC 5905 flare to partial stripping of a star or to the disruption of a substellar object, it does not help to explain the general problem of why nearby galactic nuclei are so faint. In addition to partial disruptions of the sort that might have occurred in NGC 5905, we expect total stellar disruptions in significant numbers and these should provide a significant mean mass accretion rate in galactic nuclei. Disruptions of substellar objects only add to the purely stellar disruptions considered to date. Thus, alternative ways of explaining the dimness of nearby galactic nuclei are required (Menou \& Quataert 2001; Narayan 2002), beyond the specific scenario outlined here for the giant flare of NGC 5905. Either the mass must accrete in a radiatively inefficient mode or the accretion must be suppressed or slowed down significantly.

The authors gratefully thank Stefanie Komossa for kindly providing the ROSAT data listed in Table 1 along with the conversion factors from count rate to X-ray luminosity. The authors also thank Jeremy Goodman, Bohdan Paczyński and the referee for helpful comments. LXL and RN thank the Institute for Advanced Study and the Department of Astrophysical Sciences, Princeton, for hospitality while this work was being done. LXL's and KM's research was supported by NASA through Chandra Postdoctoral Fellowship grant numbers PF1-20018 (LXL) and PF9-10006 (KM) awarded by the Chandra X-ray Center, which is operated by the Smithsonian Astrophysical Observatory for NASA under contract NAS8-39073. RN's research was supported in part by NSF grant AST-9820686. 


\section{A. Viscous Evolution of the Thin Disk Formed from Tidal Disruption}

After circularization, the fallback material from a tidal disruption event will form a torus at a radius $r \sim 2 r_{T}$. The torus will then spread out viscously and accrete onto the black hole.

The time-scale for this stage of the evolution is determined by the viscous time-scale (Cannizzo, Lee, \& Goodman 1990; Ulmer 1999)

$$
t_{\mathrm{vis}}=\frac{t_{\mathrm{Kep}}\left(2 r_{T}\right)}{\pi \alpha h^{2}} \approx 2.9 \times 10^{3} \mathrm{yr}\left(\frac{\alpha}{0.1}\right)^{-1}\left(\frac{h}{10^{-3}}\right)^{-2}\left(\frac{M_{\star}}{M_{\odot}}\right)^{-1 / 2}\left(\frac{R_{\star}}{R_{\odot}}\right)^{3 / 2}
$$

where $t_{\text {Kep }}$ is the Keplerian orbital period, $\alpha$ is the standard viscosity parameter (Shakura \& Sunyaev 1973), and $h$ is the ratio of the disk height to radius. It is easy to check that for the case of the flare in NGC 5905, where $L_{\text {peak }} \sim 10^{-3} L_{\text {Edd }}\left(M_{H} / 10^{7} M_{\odot}\right)^{-1}$, the accretion disk

would correspond to the regime of the "middle disk" in which electron scattering opacity dominates over the Kramers opacity and gas pressure dominates over radiation pressure (Shakura \& Sunyaev 1973; Novikov \& Thorne 1973; Frank, King \& Raine 1992). Then, $h$ is estimated to be

$$
h \approx 1 \times 10^{-3}\left(\frac{\alpha}{0.1}\right)^{-1 / 10}\left(\frac{\dot{M}_{2}}{10^{-3} \dot{M}_{\mathrm{Edd}}}\right)^{1 / 5}\left(\frac{M_{H}}{10^{7} M_{\odot}}\right)^{-1 / 10}\left(\frac{r}{r_{H}}\right)^{1 / 20},
$$

where $\dot{M}_{2}$ is the mass accretion rate, $\dot{M}_{\text {Edd }} \equiv L_{\text {Edd }} /\left(0.1 c^{2}\right)$, and $r$ is the radius in the disk. In equation $(\mathrm{A} 2)$ we have neglected a dimensionless factor $\sim 1$.

Inserting equation (A2) into equation (A1) and setting $r=2 r_{T}$, we obtain

$$
\begin{aligned}
t_{\text {vis } \approx} & 2.3 \times 10^{3} \mathrm{yr}\left(\frac{\alpha}{0.1}\right)^{-4 / 5}\left(\frac{\dot{M}_{2}}{10^{-3} \dot{M}_{\text {Edd }}}\right)^{-2 / 5} \\
& \times\left(\frac{M_{H}}{10^{7} M_{\odot}}\right)^{4 / 15}\left(\frac{M_{\star}}{M_{\odot}}\right)^{-7 / 15}\left(\frac{R_{\star}}{R_{\odot}}\right)^{7 / 5} .
\end{aligned}
$$

The large value of $t_{\text {vis }}$ confirms that the flare in NGC 5905 corresponded to the fallback stage, not the viscous accretion stage.

From the discussion in $\S 3$ on the fallback and viscous accretion stages associated with a tidal disruption event, the ratio of the total energy radiated during the accretion stage to the total energy radiated during the fallback stage is $2 r_{P} / r_{m s}-1$. Therefore, we can estimate the ratio of the peak of the viscous luminosity to the peak of the fallback luminosity by

$$
\frac{L_{\mathrm{vis}}}{L_{\mathrm{fb}}} \approx\left(\frac{2 r_{P}}{r_{m s}}-1\right) \frac{\Delta t_{1}}{t_{\mathrm{vis}}}
$$




$$
\approx 2.4 \times 10^{-5}\left(\frac{\alpha}{0.1}\right)\left(\frac{h}{10^{-3}}\right)^{2}\left(\frac{M_{H}}{10^{7} M_{\odot}}\right)^{1 / 2}\left(\frac{M_{\star}}{M_{\odot}}\right)^{-1 / 2}\left(\frac{2 r_{P}}{r_{m s}}-1\right)
$$

where equations (9) and (A1) have been used.

The extremely large value of $t_{\mathrm{vis}}$ and the extremely small value of $L_{\mathrm{vis}} / L_{\mathrm{fb}}$ imply that the accretion stage will never be observed in practice for the particular case of NGC 5905. [In fact, according the above estimates, one may expect a new tidal disruption before the disk evolves significantly (Syer \& Ulmer 1999; Magorrian \& Tremaine 1999).]

\section{REFERENCES}

Alexander, T., \& Kumar, P. 2001, ApJ, 549, 948

Alexander, T., \& Livio, M. 2001, ApJ, 560, L143

Ayal, S., Livio, M., \& Piran, T. 2000, ApJ, 545, 772

Bade, N., Komossa, S., \& Dahlem, M. 1996, A\&A, 309, L35

Cannizzo, J. K., Lee, H. M., \& Goodman, J. 1990, ApJ, 351, 38

Chabrier, G., \& Baraffe, I. 2000, ARA\&A, 38, 337

Choi, C. S., Dotani, T., Chang, H. Y., \& Yi, I. 2002, JKAS, 35, 1

Diener, P., Frolov, V. P., Khokhlov, A., Novikov, I. D., \& Pethick, C. J. 1997, ApJ, 479, 164

Evans, C. R., \& Kochanek, C. S. 1989, ApJ, 346, L13

Frank, J. King, A., \& Raine, D. 1992, Accretion Power in Astrophysics (Cambridge: Cambridge Univ. Press)

Frolov, V. P., Khokhlov, A., Novikov, I. D., \& Pethick, C. J. 1994, ApJ, 432, 680

Gebhardt, K. et al. 2000, ApJ, 539, L13

Hills, J. G. 1975, Nature, 254, 295

Ivanov, P. B., \& Novikov, I. D. 2001, ApJ, 549, 467

Khokhlov, A., \& Melia, F. 1996, ApJ, 457, L61

Khokhlov, A., Novikov, I. D., \& Pethick, C. J. 1993, ApJ, 418, 163 
Kim, S. S., Park, M. G., \& Lee, H. M. 1999, ApJ, 519, 647

Kochanek, C. S. 1992, ApJ, 385, 604

Kochanek, C. S. 1994, ApJ, 422, 508

Komossa, S. 2001, astro-ph/0109441

Komossa, S., \& Bade, N. 1999, A\&A, 343, 775

Lacy, J. H., Towenes, C. H., \& Hollenbach, D. J. 1982, ApJ, 262, 120

Laguna, P., Miller, W. A., Zurek, W. H., \& Davies, M. B. 1993, ApJ, 410, L83

Loeb, A., \& Ulmer, A. 1997, ApJ, 489, 573

Luminet, J. P., \& Marck, J. A. 1985, MNRAS, 212, 57

Magorrian, J., \& Tremaine, S. 1999, MNRAS, 309, 447

Marck, J. A., Lioure, A., \& Bonazzola, S. 1996, A\&A, 306, 666

Menou, K., \& Quataert, E. 2001, ApJ, 562, L137

Merritt, D., \& Ferrarese, L. 2001, ApJ, 547, 140

Narayan, R. 2002, in Lighthouses of the Universe, ed. M. Gilfanov \& R. Sunyaev, (Springer), in press (astro-ph/0201260)

Novikov, I. D., \& Thorne, K. S. 1973, in Black Holes, ed. C. DeWitt \& B. S. DeWitt (New York: Gordon and Breach), 343

Phinney, E. S. 1989, in IAU Symp. 136, The Center of the Galaxy, ed. M. Morris (Dordrecht: Kluwer), 543

Press, W. H., \& Teukolsky, S. A. 1977, ApJ, 213, 183

Rees, M. 1988, Nature, 333, 523

Renzini, A. et al. 1995, Nature, 378, 39

Ross, R. R., Fabian, A. C., \& Mineshige, S. 1992, MNRAS, 258, 189

Salucci, P., Ratnam, C., Monaco, P., \& Danese, L. 2000, MNRAS, 317, 488

Shakura, N. I., \& Sunyaev, R. A. 1973, A\&A, 24, 337 
Shimura, T., \& Takahara, F. 1993, ApJ, 419, 78

Shimura, T., \& Takahara, F. 1995, ApJ, 440, 610

Shimura, T., \& Takahara, F. 1995, ApJ, 445, 780

Syer, D., \& Ulmer, A. 1999, MNRAS, 306, 35

Ulmer, A. 1999, ApJ, 514, 180

Ulmer, A., Paczyński, B., \& Goodman, J. 1998, A\&A, 333, 379

Webbink, R. F. 1985, in Interacting Binary Stars, ed. J. E. Pringle \& R. A. Wade (Cambridge, England: Cambridge Univ. Press), 39 
Table 1. ROSAT Data on the NGC 5905 Flare

\begin{tabular}{lccll}
\hline \hline TIME $^{\mathrm{a}}$ & \multicolumn{1}{c}{ CR $^{\mathrm{b}}$} & ERR_CR $^{\mathrm{c}}$ & LUMX $^{\mathrm{d}}$ & ERR_LUMX $^{\mathrm{l}}$ \\
\hline 1990.53 & $2.2981 \mathrm{E}-1$ & $8.1771 \mathrm{E}-2$ & 1.72 & 0.61 \\
1990.54 & $2.8713 \mathrm{E}-1$ & $7.3284 \mathrm{E}-2$ & 2.15 & 0.55 \\
1990.54 & $4.1855 \mathrm{E}-1$ & $6.2263 \mathrm{E}-2$ & 3.14 & 0.47 \\
1990.54 & $4.5519 \mathrm{E}-1$ & $7.5933 \mathrm{E}-2$ & 3.41 & 0.57 \\
1990.54 & $4.1786 \mathrm{E}-1$ & $6.2403 \mathrm{E}-2$ & 3.13 & 0.47 \\
1990.54 & $5.9612 \mathrm{E}-1$ & $8.7399 \mathrm{E}-2$ & 4.47 & 0.66 \\
1990.54 & $5.4012 \mathrm{E}-1$ & $8.2004 \mathrm{E}-2$ & 4.05 & 0.62 \\
1990.97 & $<0.09$ & - & $<0.68$ & - \\
1991.04 & $<0.10$ & - & $<0.75$ & - \\
1993.55 & $7.0000 \mathrm{E}-3$ & $1.0000 \mathrm{E}-3$ & $4.00 \mathrm{E}-2$ & $5.71 \mathrm{E}-3$ \\
1996.89 & $2.9000 \mathrm{E}-3$ & $4.0000 \mathrm{E}-4$ & $1.66 \mathrm{E}-2$ & $2.29 \mathrm{E}-3$ \\
\hline
\end{tabular}

${ }^{a}$ Time of measurement in years.

${ }^{\mathrm{b}}$ Count rate in $\mathrm{cts} \mathrm{s}^{-1}$.

${ }^{c}$ Error in the count rate.

${ }^{\mathrm{d}} \mathrm{X}$-ray luminosity in units of $10^{42} \mathrm{erg} \mathrm{s}^{-1}$.

${ }^{\mathrm{e}}$ Error in the X-ray luminosity.

Note. - The data were provided by S. Komossa. The conversion from count rate to luminosity was done assuming a black-body spectrum and a Hubble constant of $H_{0}=50 \mathrm{~km} \mathrm{~s}^{-1}$ $\mathrm{Mpc}^{-1}$. For this model, $1 \mathrm{cts} \mathrm{s}^{-1}$ corresponds to $\sim 7.5 \times 10^{42}$ $\mathrm{erg} \mathrm{s}^{-1}$ during 1990 when the source was in the high-state, and $\sim 5.7 \times 10^{42} \mathrm{erg} \mathrm{s}^{-1}$ during 1993 and 1996 when the source had a flatter spectrum (S. Komossa 2001, private communication). 


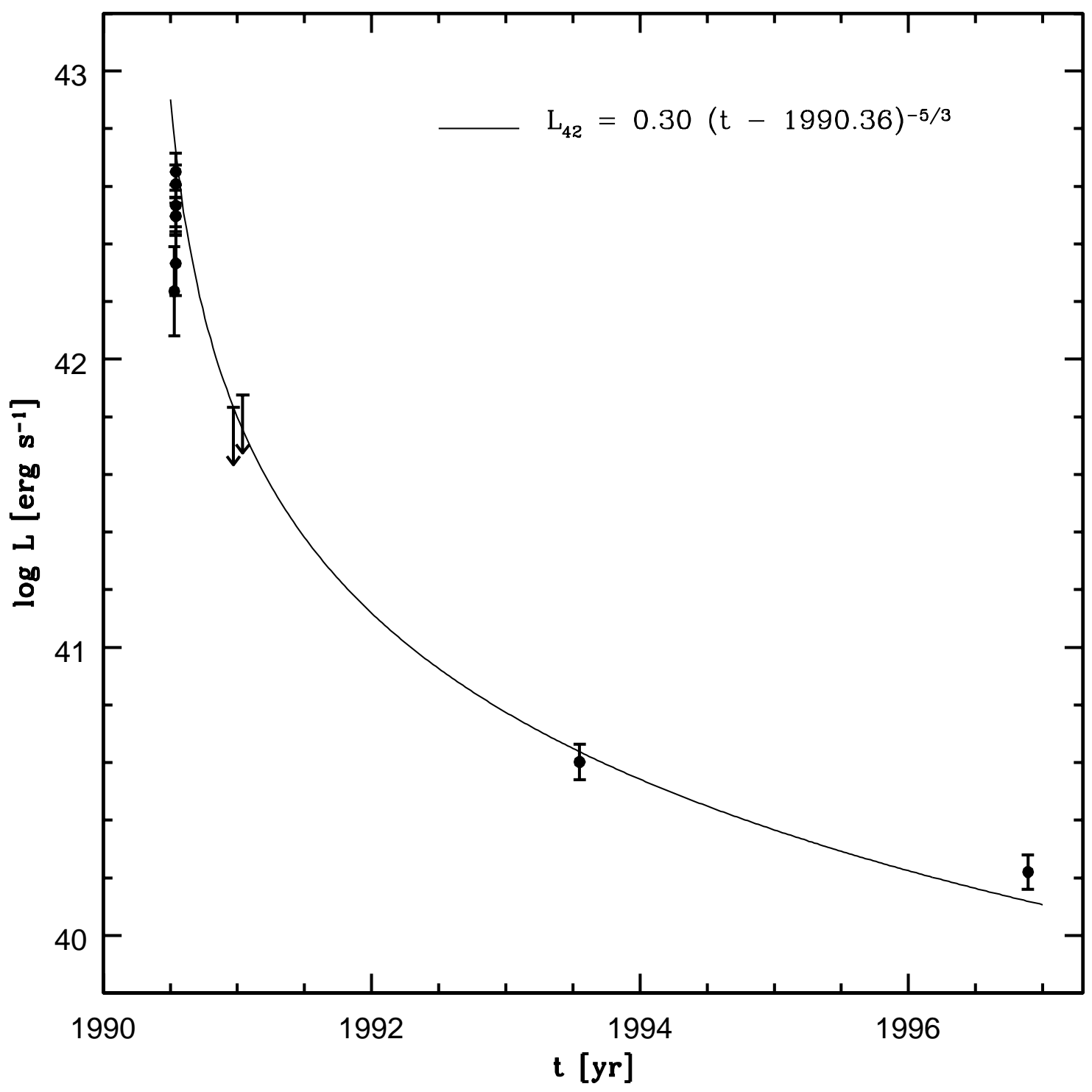

Fig. 1. - The X-ray light curve of the flare in NGC 5905 (see Table 1 for the data), fitted to a model of the form $\left(t-t_{D}\right)^{-5 / 3}$, as expected for fallback of material after a tidal disruption. 


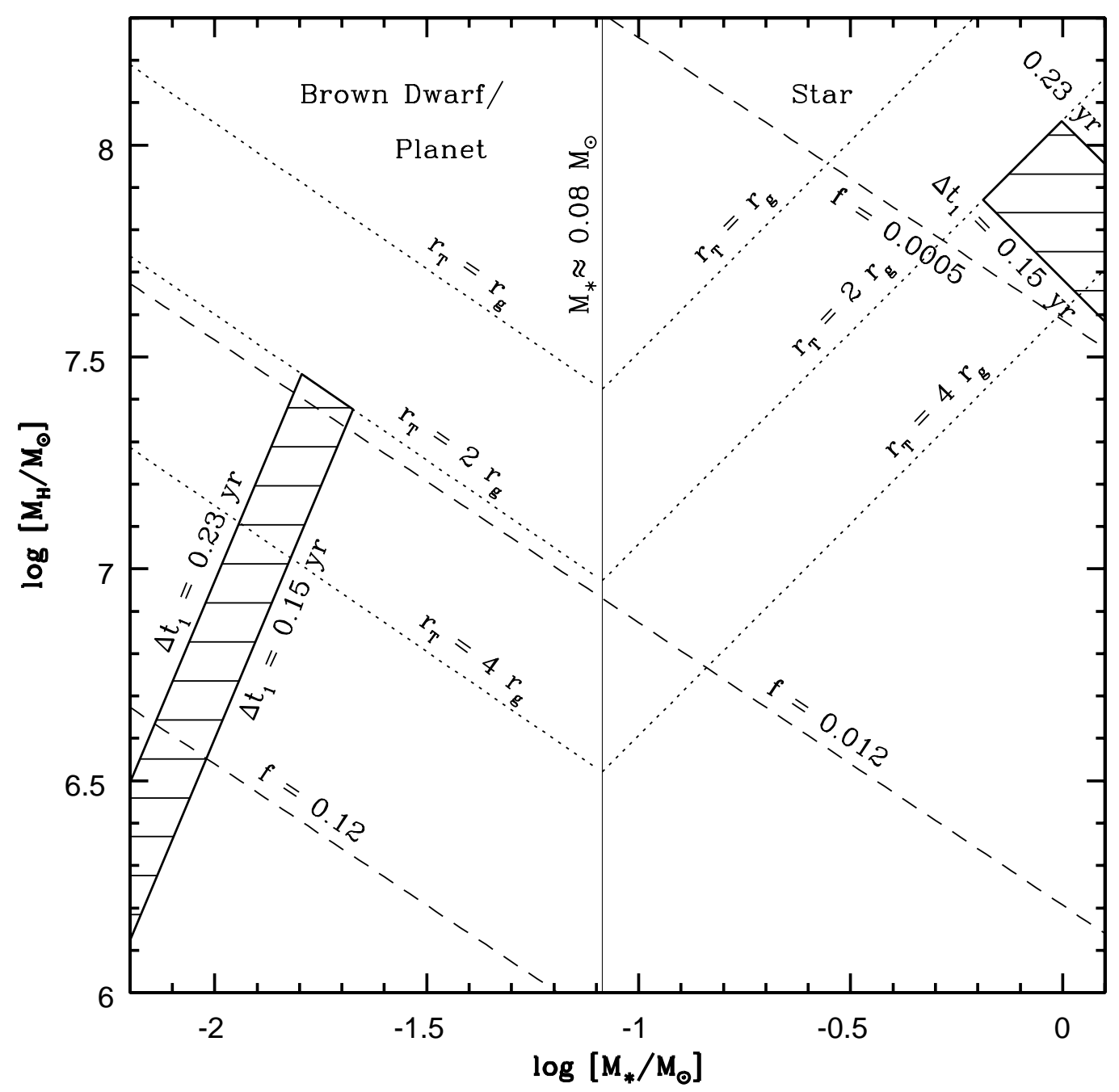

Fig. 2.- Restrictions on the mass of the black hole, $M_{H}$, and the mass of the disrupted stellar object, $M_{\star}$, derived from the X-ray flare of NGC 5905. The shaded areas show the allowed regions. It has been assumed that the star is spun up to breakup at pericenter $(k=3)$. The region to the left of the thin vertical line corresponds to brown dwarfs/planets, and the region to the right to low mass stars. The limits used are (1) $0.15 \mathrm{yr}<\Delta t_{1}<0.23 \mathrm{yr}$, where $\Delta t_{1}$ is the time for the most bound disrupted material to fall back to pericenter; and by (2) $r_{T}>2 r_{g}$, where $r_{T}$ is the tidal disruption radius (assumed to be equal to the pericentric radius) and $r_{g} \equiv G M_{H} / c^{2}$. For comparison, lines corresponding to $r_{T}=r_{g}$ and $r_{T}=4 r_{g}$ are also shown. The dashed lines correspond to equation (16) for various values of the fraction $f$ of the mass of the disrupted star that falls back. 


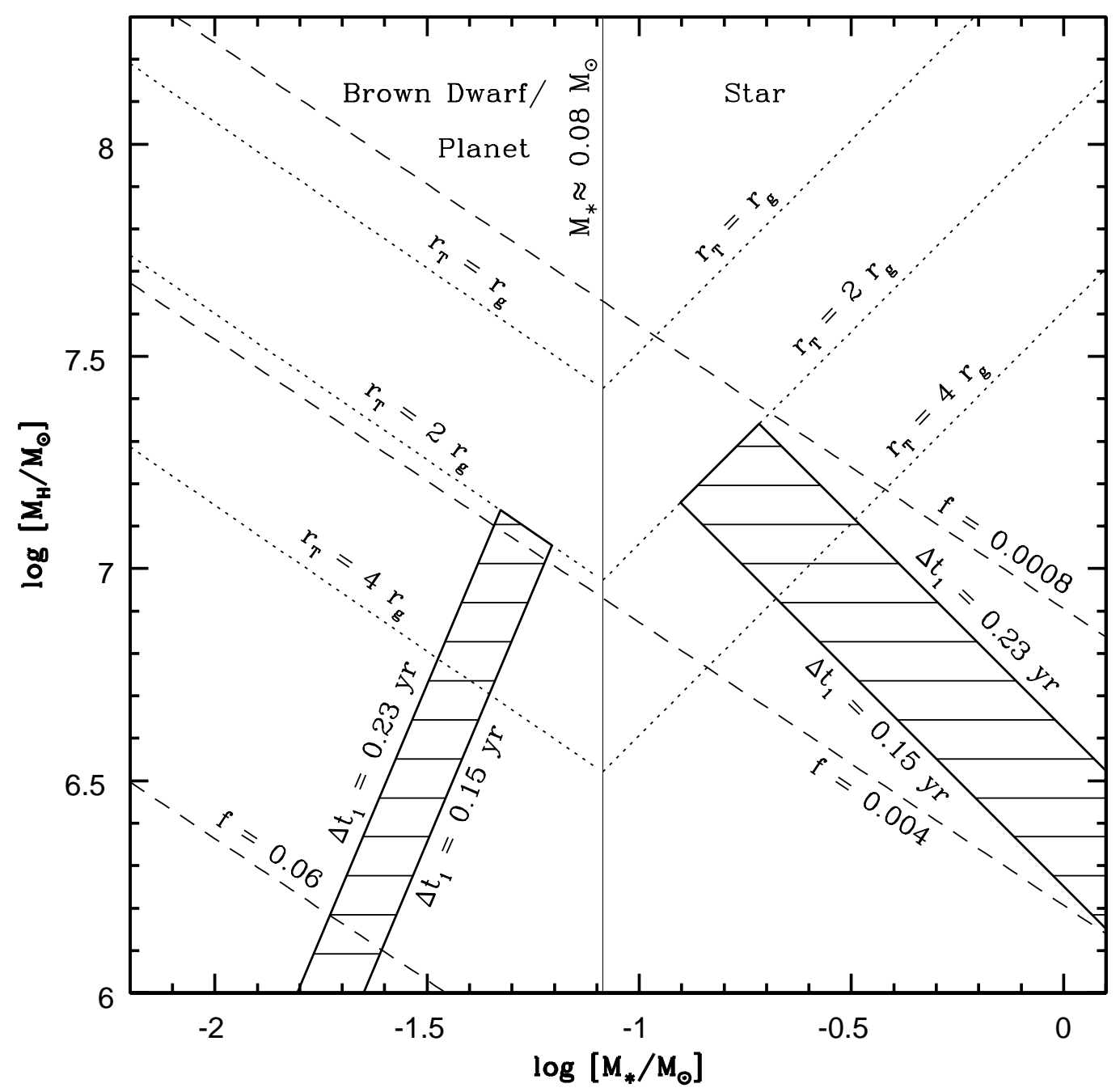

Fig. 3.- Same as Fig. 2, but assuming that the star experiences negligible spin-up $(k=1)$. 


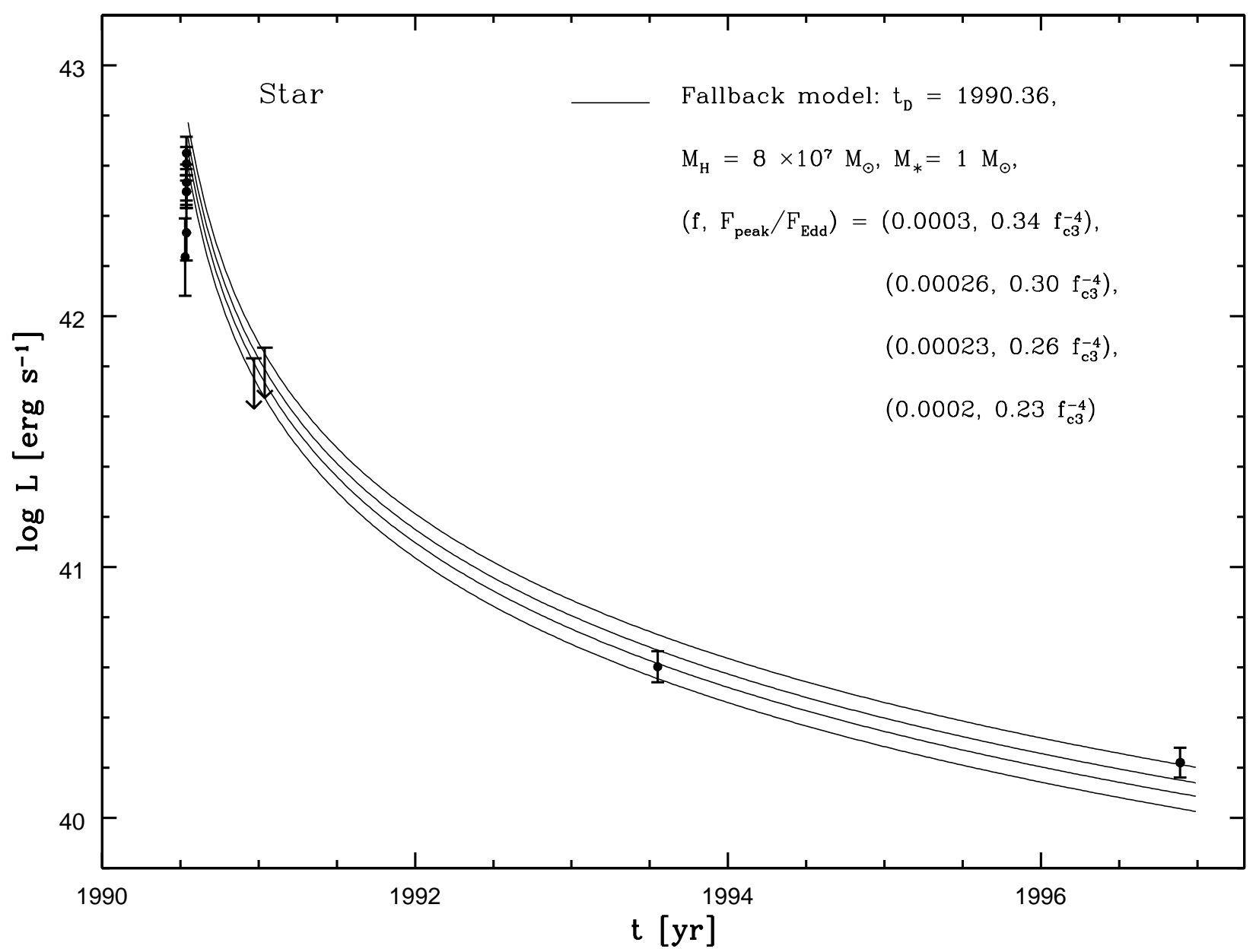

Fig. 4. - Comparison of light curves predicted by the fallback model with data: the disrupted object is assumed to be a low mass star. The parameter $f$ is the ratio of the accreted mass to the total mass of the star, $F_{\text {peak }} / F_{\text {Edd }}$ is the ratio of the peak flux $\equiv L_{\text {peak }} /\left(\pi R_{X}^{2}\right)$ to the Eddington flux $\equiv L_{\text {Edd }} /\left(4 \pi r_{T}^{2}\right)$, where $f_{c 3} \equiv f_{c} / 3$. Four models are shown, each with the values of $f$ and $F_{\text {peak }} / F_{\text {Edd }}$ indicated (from top to bottom). 


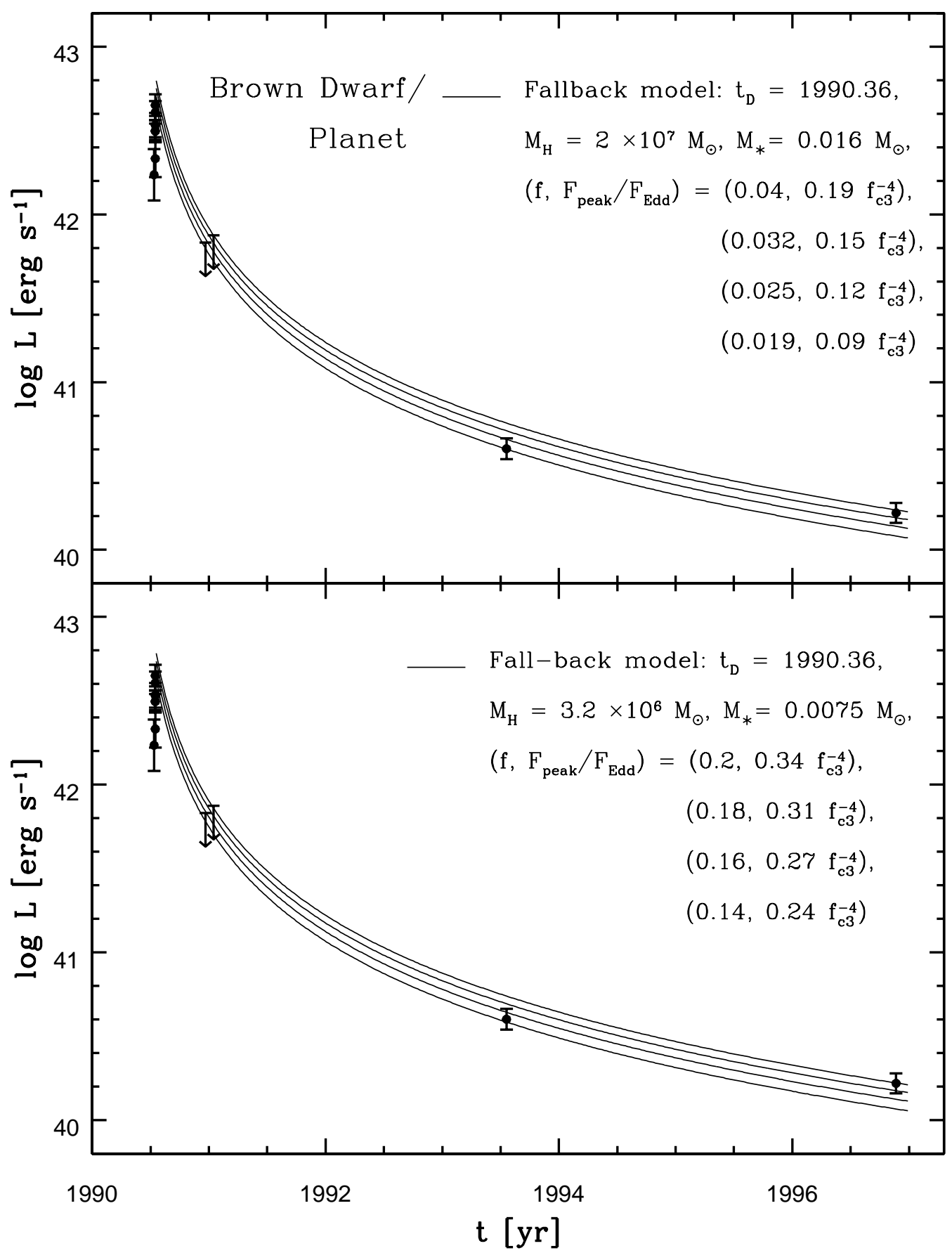

Fig. 5.- Similar to Fig. 4, but assuming that the disrupted object is a brown dwarf or a planet. Two cases are shown: $M_{H}=2 \times 10^{7} M_{\odot}$, brown dwarf of mass $M_{\star}=0.016 M_{\odot}$ (upper panel); and $M_{H}=3.2 \times 10^{6} M_{\odot}$, planet of mass $M_{\star}=0.0075 M_{\odot}$ (lower panel). 